\title{
EL BAILE Y LA MÚSICA POPULARES \\ COMO ESPACIO DE FORMACIÓN EN VIRGILIO MORA: ALGUNOS EJEMPLOS DE LA DISTANCIA DEL ÚLTIMO ADIÓS ${ }^{l}$
}

\author{
Jorge Chen Sham
}

\begin{abstract}
RESUMEN
Se estudian dos relatos de La distancia del último adiós (1995), "Figueredo" y "Excelente para el cutis", en los que el costarricense Virgilio Mora desarrolla magistralmente la significación que poseen el baile y la música populares en la conformación de la identidad del sujeto. Se trata de valorar estas prácticas de socialización en tanto ritos de pasaje o de iniciación de sus protagonistas en los que la primacía de la vivencia los hace fundamentales en la constitución y en el desarrollo de los individuos.

Palabras clave: Virgilio Mora, música y baile populares, narrativa costarricense, vivencia bajtiniana.
\end{abstract}

\begin{abstract}
This work investigates two relationships of La distancia del último adiós (1995), "Figueredo" and "Excelente para la piel" in which the Costa Rican Virgilio Mora skillfully develops the meaning that is represented by the dance and popular music in the development of the identity of the individual. He evaluates the value of these socialization practices in as much as rites of passage or initiation of his protagonists in that the significance of the experience is a fundamental one in the constitution and development of the individuals.

Key words: Virgilio Mora, popular music and dance. Costa Rican narratives, experiences bajtiniana.
\end{abstract}

La narrativa deVirgilio Mora ha sido caracterizado por María Amoretti Hurtado dentro de una construcción de una subjetividad y una conciencia autobiográfica (2001: 40). Para desarrollar esta narrativa del self o de la identidad del sujeto, el escritor costarricense se vale de un tipo de relato de repetición rememorativa ${ }^{2}$ en el que existe una fascinación/ aversión por reconstruir los lugares y los acontecimientos que marcan su infancia y juventud de un personaje maduro. Surgen, entonces, el espacio de la Costa Rica de los años 50 y 60 y

\footnotetext{
Jorge Chen Sham. Profesor de la Escuela de Filología, Lingüística y Literatura, Universidad de Costa Rica. San Pedro, San José, Costa Rica.

Correo electrónico: jorgechsh@yahoo.com
}

Recepción: 5- 6- 2009

Aceptación: 27- 6- 2009 
ese marcado interés del proyecto ideológico de Mora por aproximarse compulsivamente a sus olores, sabores, sonidos, colores, fragancias, comidas, etc., tal vez para marcar esa necesidad de no olvidar ante la distancia geográfica y temporal. Esta percepción sensorial, porque se vale de la primacía de los sentidos para captar una realidad y plasmarla fotográficamente para el lector, hace que Virgilio Mora no escatime esfuerzos en crear un ambiente propicio para crear un ambiente de época y un tipo de narración cuya clave está en el ejercicio de la memoria.

No es inocente que los relatos cortos en estudio se encuentren en una colección cuyo título sea La distancia del último adiós (1995). El regreso a la infancia y a la juventud es el núcleo diegético que se desarrolla repetitivamente, en donde la fascinación nostálgica por el pasado revelaría no tanto el intento de recuperar ese paraíso perdido como generalmente lo interpretamos dentro del motivo del regressus ad uterum, como el regreso de ese pasado que interpela y obliga al personaje narrador a incorporar sus recuerdos ante un tiempo cronológico que hace sus estragos sobre la memoria. De ahí que la experiencia narrativa retome estos recuerdos y les de una significación dentro de novela de formación de personaje y el desarrollo de una "conciencia individual". A ello corresponde la estructura de aprendizaje de la novela de formación, en donde la educación se relaciona con esa capacidad que posee el narradorprotagonista de analizar su biografía personal en tanto experiencia cognoscitiva y estética, es decir, en tanto vivencia que involucra "la contemplación", tal y como lo explica Bajtín:

\footnotetext{
El objetivo, el sentido y el propósito dejan de regir valorativamente y se convierten sólo en características de la dación autónoma de la vivencia. La vivencia es la huella del sentido en el ser, es un destello sobre el ser, desde su interior la vivencia no vive por sí misma sino por este sentido que se encuentra y se capta afuera, porque cuando la vivencia no capta el sentido es que simplemente no existe; la vivencia representa la relación con el sentido y el objeto y fuera de esta relación no existe para sí [...] (1982: 105).
}

La necesidad de sentido es lo que desemboca en esta capacidad de la vivencia para plasmarse estéticamente, dentro de un contexto valorativo o significativo para el sujeto. En el caso de la vivencia de la infancia, se trata de la posibilidad de modelar esa experiencia como necesidad de hacer hablar ese pasado del adulto que fue niño alguna vez. Por esa razón, transformado en aprendizaje y en un proceso de desarrollo del personaje, ese pasado conduce a que la indagación del mundo de la niñez (también el de la adolescencia y el de la juventud) se plantee como indagación de "saber la infancia" y de hacer "hablar la niñez" (Schatzman 2003: 24-6), en la que están inscritas todas las posibilidades y todas las alternativas del protagonista.

Lo anterior nos obliga a considerar la toma de conciencia del individuo con el desarrollo de una forma novelesca, capaz de representar la educación y el desarrollo individual del protagonista. Para ello, Virgilio Mora se vale de la cultura popular y, más específicamente, de la música y del baile. Vista de esta manera, la transmisión de esta cultura popular debe plantearse en forma de ritos y espectáculos en los que los procesos de sociabilización se asocian con los espacios públicos, abiertos y de celebración festiva colectiva. En su fundamental L'oeuvre de François Rabelais et la culture populaire au Moyen Age et sous la Renaissance, Mijail Bajtín ${ }^{3}$ desarrolla los alcances de estos planteamientos en términos de unas relaciones humanas radicalmente distanciadas de una cultura oficial (1970: 13) y, de este modo, Bajtín plantea la existencia de formas de expresión y de comunicación ligadas a lo cómico y al juego del espectáculo (1970: 15), es decir, al carnaval que desinhibe y deja salir lo reprimido.

El baile y la música, propios de la fiesta carnavalesca, forman parte de ese espectáculo social que dura el tiempo que se asiste a su puesta en escena; de eso dan cuenta esos salones populares de baile en el que los asistentes/actores viven y se recrean en tanto comulgan y se 
identifican con una vivencia ${ }^{4}$. El baile y la música se vuelven instante; son del tiempo de lo efímero y pueden verse como simulacrum. La fiesta surge en toda su expresión ritual, como ya lo señaló María Amoretti para ese texto fundacional de la narrativa moriana que es Cachaza (1977), en donde la fiesta carnvalesca constituye, para ella, estructura profunda y motivo textual (1989: 127), con lo cual esta novela termina por hacer del carnaval "manifestación lúdica del texto, es decir, representación del espectáculo” (Amoretti 1989: 128).

En Virgilio Mora, la vivencia expresa este comportamiento carnavalesco y lúdico, por cual entraña un alto contenido festivo y perentorio de lo que se capta en un instante; pero vuelve a la memoria en forma de un sonido o de un olor,por ejemplo. Por lo tanto, esta se moldea en forma de una experiencia, es decir, en forma de un hecho que tiene función y sentido en la conformación de la identidad del personaje y, por lo tanto, necesaria a la formación personal. La primacía de esta experiencia, en tanto que ella es el fundamento de la socialización del sujeto, da cuenta una serie de relatos de Mora en los que el protagonista es un adolescente o un joven en formación. Pero tal experiencia desemboca, al mismo tiempo, en la afirmación de la importancia del desarrollo de las facultades del baile en el proceso de socialización y de la aceptación social del individuo. En el contexto de los adolescentes, el baile y la música se transforman en prácticas de sociabilidad que las relaciones sociales imponen para poder cohesionar a los individuos y, de este modo, ofrecerles la posibilidad de comunicar, negociar y adecuar sus comportamientos a códigos socio-culturales y ritos de iniciación. Cada sociedad posee una serie de ritos y de actos que le permiten un mínimo de armonía en la interacción comunicativo-social, nos plantea Catherine Kerbrat-Orecchioni (2004: 39); de esta manera, la importancia del baile en los aprendizajes sociales de los adolescentes es innegable. De los bailes estudiantiles, a las fiestas de adolescentes, pasando por los bailes en los clubes o centros sociales, la música y el baile se perfilan con esa connotación de "pasaje" y "ritual social", amén de que permiten socializar y desinhibir a los individuos, en contextos en donde el salón, el baile, la luz, el contacto físico y el licor desencadenan la fiesta.

La narrativa de Virgilio Mora da cuenta de la importancia de los bailes y la música popular como ritos de iniciación y su "valoración objetiva" en el sentido bajtiniano, es decir, colocarse afuera y en la totalización predeterminada (Bajtín 1982: 106). Veamos varios ejemplos en La distancia del último adiós. En "Figueredo", el protagonista se presenta como un joven del Liceo de Costa Rica, que va a fiestas en las que toca una orquesta. Nos remontamos a lo que se denomina la etapa de oro de la música popular de los 50 y 60 en América Latina, en la que dominan los boleros, los mambos y otros ritmos populares; espacio que, para el caso costarricense ha recreado Mario Zaldívar, quien recoge entrevistas y opiniones de los que vivieron esa época de oro de la música popular (2003). Para el joven liceísta, preocupado en sus relaciones con las féminas y con una sexualidad exacerbada, los bailes le permiten intimar con las "hembrillas" (163) en un contexto en el que se quiebran la rigidez de las normas sociales y se permite el contacto y la relación entre personas de diferente sexo, o de estratos también diversos. Veamos lo que dice el protagonista a su amigo Figueredo para convencerlo de que lo acompañe al próximo baile:

En la tarde fui al baile del Señoritas en el anexo del Teatro Nacional. Ahí tampoco te vi. Estás hecho un ermitaño, güevón. Estuvo muy bueno también. Me encontré con Angie (Angie es la semita que me gusta. No me animé a sacarla. Andaba con un montón de paisanas). Tocaron una canción que me gustó mucho, "Ballerina” o "Dance Ballerina”, con Bob Monroe. Me bailé esa pieza dos veces con la misma hembrilla, una muchacha que vive en Barrio México, compañera de Flor Martínez (163). 
Desde este punto de vista, el protagonista no puede todavía comprender cómo Figueredo se resiste a acompañarlo y, menos aún, que deje de aventurarse a un baile por estar ennoviado, cuando lo importante es socializar y no encerrarse en el "yugo" de la relación formal del noviazgo:

\footnotetext{
Figueredo es caso perdido, igual que el Negro Chavarría, igual que Gómez; enamorados, viven solo para sus novias, desde que conocieron a sus respectivas hembrillas, no hacen más que "marcar" a todas horas. "Deberíamos ir a esa fiesta, güevón. Dicen que va a estar muy buena, con la Orquesta Murillo nada menos. Ocho pesos la entrada. A lo mejor nos podemos colar. ¿Por qué no te animás? (163).
}

Para el protagonista de "Figueredo", la adolescencia es la etapa de ir a los bailes y de enamorarse de las muchachas sin tener ningún compromiso serio. Se podría concebir como la época de la aventura y del disfrute, así como del descubrimiento de la sexualidad; por eso no puede entender que sus compañeros tengan novia y ya no estén interesados en correr los bailes con la libertad que el noviazgo elimina. Su poder de convencimiento es nulo con Figueredo y fracasa el intento de convencer a su mejor amigo de que lo acompañe al baile del próximo sábado en La Casa España. Contigüidad obliga, la provocación de Lev, quien viene a burlarse del protagonista y de Figueredo pues su equipo de futbol viene de perder, complica el sentimiento de que las cosas le están saliendo mal. En efecto, el equipo de la capital, el Deportivo Saprissa, viene de perder 2 por 0 contra su archirrival de siempre, la Liga Deportiva Alajuelense. La respuesta emotiva del protagonista es inmediata: "Me altero, súbitamente me siento más caliente. ¡Qué manera de empezar la semana!; y esto es apenas el comienzo [...]” (164).

Desde la perspectiva del adolescente, la significación de los hechos que conforman la existencia posee otra dimensión; su mundo se compone de bailes y de su afición al futbol, de manera que su vida se organiza otorgándole significación de primer orden a aquellas actividades que permiten socializar e identificarse colectivamente con grupos humanos. Con su maestría narrativa, Virgilio Mora nos narra la importancia, para el protagonista del relato, de que Figueredo, su mejor amigo, lo acompañe al baile; se trata de una etapa de la vida en la que se necesita la aceptación y el reconocimiento de los otros. Pero también sugiere, en la contigüidad de los dos acontecimientos, una relación que solamente se explicitará en otro relato la relación entre el baile y el deporte y su relación en el aprendizaje sexual.

Por el contrario, en un contexto del joven que debe ganar experiencia de la vida y desenvolverse en los roles que la sociedad exige al "macho" latino, "Excelente para el cutis" nos sitúa en la valoración del baile y de la música en esta formación para la vida, cuando el narrador nos presenta a Mireya, la mujer recostada sobre la rocola, como "[su] maestra de baile" (153) y nos recrea ese mundo de cantinas y salones de baile en el que se termina aceptando, como dice el narrador con ironía, ese deporte social que se juega en tales espacios de convivencia; afirma "a no ser que bailar y beber al mismo tiempo sea [considerado] un deporte" (153). Se trata, en "Excelente para el cutis", de un ambiente inundado de música de boleros y de mambos, en donde surge la figura de Mireya, la cual se contornea en sus movimientos, que el narrador describe con gran sensualidad cuando baila con un joven estudiante de medicina:

[...] ahora se dirige a la rocola desde donde lo llama ella con un gesto, con un movimiento del índice que invita a bailar, a refocilarse, a escarbarle a la mujer la entrepierna con la pierna derecha a los acordes de Patricia, otro paso hacia atrás, dos tres... cuatro pasos hacia atrás muy quebrados, moviendo mucho la cintura con un movimiento que remeda la cópula en posición erecta, una forma de bailar para la que Mireya está bien dotada, unas buenas caderas, unas magníficas piernas y una forma desvergonzada de agarrarse a panzazos, a caderazos con su pareja [...] (154). 
El narrador protagonista no solo observa con detenimiento el baile y el acoplamiento de los cuerpos en la pista de baile, sino también, en tanto voyeur, se regodea de esa escena que describe sensualmente con un lenguaje bien explícito. No es casual, por ello, que el protagonista se sumerja, como él manifiesta, en "un aspecto de la vida social de San José que no conocía" (155); se trata del San José de salones de baile, los llamados "clubes sociales" en los que se rompían las barreras sociales y se vivía el instante del ceremonial del carnaval, como lo apunta el mismo protagonista:

[...] los domingos por las tardes se mezclan la clase media con las clases más bajas, donde estudiantes, internos y residentes de hospitales, empleados de banco, bailan con cocineras, donde prostitutas se olvidan de su profesión para entregarse en cuerpo y alma al baile, donde todos, sin distinción de clases, mientras dura la música, nos sentimos especiales... (155).

Como indica Bajtín, si el carnaval ignora toda distinción, su carácter festivo destruye las distinciones entre actores y espectadores (1970: 15), elimina las fronteras y los transforma todos en parte del mismo espectáculo social que dura mientras se realice provisoriamente la fiesta. El baile y la música del salón tiene aquí esa función social; pero desde el punto de vista de la experiencia del individuo, tiene otra significación: le permiten al protagonista descubrir un mundo desconocido a sus ojos y una realidad social en la que ahora se inserta explorándola. Dicho de otra manera, la música popular y el baile funcionan como el desencadenante de un aprendizaje personal, gracias al cual descubrirá otra faceta de su sexualidad, cuando se liga a Mireya. Rápidamente se inserta en este mundo proclive al disfrute sin barreras, un mundo catalogado de bajos fondos y visto desde la óptica picaresca, cuando se le abren todas las puertas:

[...] el primer lugar en mi vida donde puedo tomar una birra e irme sin pagar, un lugar desde el que no me toman más de cinco minutos para llegar a la entradita de la casa de Mireya, una entradita oscura; ahí ella me hace algo que yo no sabía que hacían las mujeres, algo que ella hace con la mayor voluntad del mundo porque dice que es excelente para el cutis... (155-6).

El descubrimiento de una sexualidad libre de las prohibiciones sociales se relaciona con lo que Mireya, su maestra de baile y de algo más, como lo sugiere el texto, le enseña aquí al protagonista. Narrado con una gran maestría en un desenlace sorpresivo, el relato de Mora se nutre de un consabido de la doxa popular en cuanto al sexo como podría ser el semen y la eyaculación del hombre ${ }^{5}$, al mismo tiempo, juega con él para desencadenar este final en el que se explota la sexualidad y lo genital con apenas insinuarlo. El rito de iniciación se insinúa con ese acto en contra de las leyes sociales ("puedo tomar una birra e irme sin pagar") y la exploración de lo prohibido con el pasaje a un lugar recóndito ("una entradita oscura"), en donde Mireya se erige maestra de un saber femenino ("ahí ella me hace algo que yo no sabía que hacían las mujeres"). La iniciación sexual del joven se anuncia con esa sorpresa que produce la ironía del cierre del relato en forma de una sinécdoque, que solamente es significativa para quien sepa descodificar el acto de sexo oral que la solícita Mireya le brinda al protagonista. Pero lo magistral del relato de Mora es que no haga tanto hincapié ni en la erección ni en la eyaculación, sino en la perspectiva de quien goza la gratificación, según la perspectiva de Román Gubern, "lo que puede ocurrir en la actividad sexual de las mujeres" (1989: 164), quien interpretaría el punto de vista del relato de Mora desde la vivencia de lo auténtico ${ }^{6}$.

Por lo tanto, ya sea en la adolescencia, ya sea en la juventud, los relatos de Mora nos presentan el baile y la música, insertos dentro de una experiencia formativa para el individuo, eso es evidente; pero lo es a partir de las sensaciones que el adolescente o el joven deben 
experimentar en su doble acepción de comprobar y ensayar por un lado, y por otro, de sentir y ser afectado por su entorno social, más allá de los conocimientos adquiridos en la educación formal. Cuando Bajtín nos hablaba de la vivencia, la presentaba como esa valoración estética de quien observa a la distancia la importancia y la significación de su pasado; en los dos relatos de La distancia del último adiós, este aserto se cumple en un escritor que hace del espacio de la música y bailes populares elementos significativos del aprendizaje de sus protagonistas. Con ello, Virgilio Mora tiene el encanto y la maestría de sumergirnos en esos sonidos musicales y movimientos del cuerpo que nos invitan al regodeo nostálgico de un tiempo transcurrido pero que se instancializa, se vuelve presente en la experiencia de sus personajes de ficción.

\section{Notas}

1. Se trata de una versión corregida y aumentada de la ponencia que presenté en el XXX Simposio Internacional de Literatura "La literatura Iberoamericana y otras artes", celebrado en agosto del 2008 en la Prefectura de Porto Alegre (Brasil).

2. Retomo el término de De Feo, para quien esta repetición compulsiva está en relación con la asunción del pasado y de la manera en que , por medio de la memoria, el sujeto quiere apropiarse de él (2007: 29).

3. Utilizo la grafía española del nombre del crítico ruso, aunque utilizo la traducción francesa de su obra.

4. Lo que ocurre especialmente con esta función en la que memoria narrativa se regodea recordando esa música de los 50 y 60 y la instancia narrativa la recuerda, de rebote, con nostalgia.

5. Lo que en el lenguaje popular recibe el nombre de la "leche". Otros consabidos en materia sexual sería que la masturbación masculina excesiva es nociva.

6. Lo contrario, apuntaría Gubern, sería la simulación del acto pornográfico, centrado en la apariencia de la interpretación, allí el sexo no sería vivencia, es decir, experiencia.

\section{Bibliografía}

Amoretti Hurtado, María. 1989. Introducción al socio-texto: A propósito de “Cachaza”. San José: Editorial de la Universidad de Costa Rica.

2001““Por qué querer un hijo doctor? Análisis de Los problemas del gato”. Revista de Filología y Lingüística de la Universidad de Costa Rica. 27 (1): 39-52.

Bajtín, Mijaíl. 1970. L’oeuvre de François Rabelais et la culture populaire au Moyen Age et sous la Renaissance. París: Éditions Gallimard.

1982. Estética de la creación verbal. México, D.F.: Siglo Veintiuno Editores. 
De Feo, Miguel Ángel. 2007. "El acoso de las fantasías: La pulsión atracción-repulsión en ¿Quién inventó el mambo? de Rosa María Britton”. Rosa María Britton ante la crítica. Humberto López-Cruz (ed.). Madrid: Editorial Verbum, 29-45.

Gubern, Román. 1989. “El discuso fílmico y la intimidad”. De la intimidad. Carlos Castilla del Pino (Comp.). Barcelona: Editorial Crítica, 151-72.

Kerbrat-Oreacchioni, Catherine. 2004. “Es universal la cortesía?”. Pragmática sociocultural: Estudios sobre el discurso de cortesía en español. Diana Bravo y Antonio Briz (eds.). Barcelona: Editorial Ariel, 39-65.

Mora, Virgilo. 1995. La distancia del último adiós. San José: Editorial de la Universidad de Costa Rica.

Schatzman, Morton. 2003. El asesinato del alma: La persecución del niño en la familia autoritaria. $17^{a}$ edición. México, D.F.: Siglo Veintiuno Editores.

Zaldívar, Mario. 2003. Costarricenses en la Música (Conversaciones con protagonistas de nuestra música popular, 1939-1959). San José: Departamento de Publicaciones del Ministerio de Cultura, Juventud y Deportes. 
